\title{
Aesthetic outcomes and complications following post-mastectomy radiation therapy in patients undergoing immediate extended latissimus dorsi flap reconstruction and implant insertion
}

\author{
Yun Hyun Kim ${ }^{1 \wedge}$, Joon Seok Lee ${ }^{1}$, Jongmoo Park², Jeeyeon Lee ${ }^{3}$, Ho Yong Park ${ }^{3}$ Jung Dug Yang ${ }^{1 \wedge}$ \\ ${ }^{1}$ Department of Plastic and Reconstructive Surgery, School of Medicine, Kyungpook National University, Jung-gu, Daegu, Korea; ${ }^{2}$ Department \\ of Radiation Oncology, Kyungpook National University Chilgok Hospital, Buk-gu, Daegu, Korea; ${ }^{3}$ Department of Surgery, School of Medicine, \\ Kyungpook National University, Jung-gu, Daegu, Korea \\ Contributions: (I) Conception and design: YH Kim, JD Yang; (II) Administrative support: J Park, HY Park, JD Yang; (III) Provision of study materials \\ or patients: YH Kim, JS Lee, J Lee; (IV) Collection and assembly of data: YH Kim, JS Lee; (V) Data analysis and interpretation: YH Kim, JD Yang; \\ (VI) Manuscript writing: All authors; (VII) Final approval of manuscript: All authors. \\ Correspondence to: Jung Dug Yang, MD, PhD. Department of Plastic and Reconstructive Surgery, School of Medicine, Kyungpook National \\ University, 130 Dongdeok-ro, Daegu 41944, Korea. Email: lambyang@knu.ac.kr.
}

\begin{abstract}
Background: Complications of radiotherapy after implant-based reconstruction include capsular contracture development, seroma formation, and reoperation for implant removal or replacement. However, there is a lack of studies regarding aesthetic outcomes and complication rates following radiation therapy among patients undergoing latissimus dorsi (LD) flap-based reconstruction with implant insertion for volume shortage. The present study aimed to evaluate clinical and aesthetic outcomes of post-mastectomy radiation therapy (PMRT) among patients receiving both LD flap reconstruction and implant insertion.

Methods: This study comprised 66 patients who underwent mastectomy and breast reconstruction between March 2014 and July 2019. Patient demographics and outcomes were compared among patients who did and did not receive PMRT. Aesthetic outcomes were compared using gross photographs. The incidence of complications, including seroma formation, flap necrosis, nipple-areola complex necrosis, hematoma development, and capsular contractures, was compared between groups.

Results: No differences in aesthetic outcomes using gross photos during outpatient follow-up were observed between the radiation and control groups. No significant difference in the frequency of complications was observed between groups.

Conclusions: The use of implants and LD reconstruction are inevitable in a proportion of patients due to a lack of LD flap volume. For these patients, PMRT could be safe treatment option if the necessary precautions are implemented.
\end{abstract}

Keywords: Latissimus dorsi (LD); silicone implant; post-mastectomy radiation; breast reconstruction

Submitted Apr 05, 2021. Accepted for publication Jun 22, 2021.

doi: $10.21037 / g s-21-219$

View this article at: https://dx.doi.org/10.21037/gs-21-219

^ ORCID: Yun Hyun Kim, 0000-0002-9137-0909; Jung Dug Yang, 0000-0002-9040-4724. 


\section{Introduction}

Breast reconstruction after mastectomy or breastconserving surgery for breast cancer can be divided into autologous versus implant-based reconstruction. The most common method of autologous-based reconstruction is the use of a latissimus dorsi (LD) flap, which has proven to be a safe procedure with optimal aesthetic outcomes in previous studies. However, deformities and asymmetry can occur in cases where there is a lack of flap volume for LD flap reconstruction. In such cases, previous studies have demonstrated that supplementation with optimal implant volume can provide a safe method of obtaining acceptable aesthetic outcomes (1).

Radiotherapy (RT) is widely used as part of breastconserving treatment for early breast cancer (2). Indications for RT have continued to expand in patients who have undergone a mastectomy (3), and RT has been demonstrated to improve oncologic outcomes in patients with a node-positive status following a mastectomy. As a result, RT is increasingly recommended as adjuvant therapy for patients undergoing breast reconstruction (4).

RT has been shown to be associated with lower patient satisfaction with aesthetic outcomes and an increased rate of reconstruction complications such as capsular contractures, seroma formation, and reoperation for implant removal or replacement (5). Previous studies have posited that irradiation of healthy tissue decreases vascularity leading to a decrease in tissue oxygenation, often resulting in fibrosis (6).

However, there is a lack of studies evaluating aesthetic outcomes and complication rates following postoperative radiation therapy in patients undergoing LD flap reconstruction surgery using an additional implant for insufficient volume. A previous study reported that LD flap supplementation during second-stage surgery could provide healthy tissues in patients with complications after implant-based reconstruction or post-mastectomy radiation therapy (PMRT). This method resulted in a reduction in complications and improved patient satisfaction (7). A separate study demonstrated high patient satisfaction with aesthetic outcomes and a lower rate of complications, such as fat necrosis, with $\mathrm{LD}$ reconstruction compared to the use of other autologous tissues, even when the LD flap is irradiated (6). A further study demonstrated that LD flaps have a low ratio of fatty tissue and are more resistant to radiation therapy compared to autologous abdominal wall flaps (8). These results indicate radiation therapy after breast construction with an LD flap can improve patient outcomes.

We hypothesized radiation has no effect on aesthetic outcomes or complication rates in patients undergoing LD flap reconstruction plus implant surgery. We, therefore, evaluate clinical and aesthetic outcomes in 66 patients who underwent LD flap reconstruction with implant insertion and received PMRT.

We present the following article in accordance with the STROBE reporting checklist (available at https://dx.doi. org/10.21037/gs-21-219).

\section{Methods}

This was a retrospective cohort study comprising 66 patients who underwent mastectomy and LD flap breast reconstruction with silicone implant insertion between March 2014 and July 2019. All patients underwent a total mastectomy with immediate breast reconstruction. The surgeries were performed by the same breast surgeon, and reconstructions were performed by another plastic surgeon.

\section{Extended LD flap reconstruction and implant insertion}

Approaching through the deep fat layer, dissection was started from the anterior border of the LD muscle originating from the external surface of the third or fourth most inferior rib. Dissection was then continued to the paravertebral area of the LD posterior border, to the overlapping area with the external oblique muscle on the inferior side, and to the bifurcation between the thoracodorsal artery and the LD branch of the superior area, thereby isolating and elevating the LD flap. The subcutaneous layer of the flap was then tunneled and elevated before being positioned close to the breast. The skin of the LD flap was de-epithelized to fit the defect site. The use of an LD flap alone can lead to severe asymmetry and inadequate coverage due to the large defect created. We, therefore, inserted silicone implants under $400 \mathrm{cc}$ negative pressure drainage superior and inferior to the LD flap. Irrigation was performed using a solution containing an antibiotic agent. After fixing the area to be placed on the superior aspect of the LD flap to the pectoralis major muscle using 2-0 vicryl (Ethicon, Somerville, NJ, USA), a silicone implant was positioned underneath the LD flap and anterior to the pectoralis major muscle. The LD flap was then fully covered to prevent implant exposure. We did not perform fixation of the area to be placed in the inferior part 
of the LD flap. Surgery was completed with layered closure.

\section{Radiotherapy}

All patients who received RT underwent computed tomography simulation in the supine position. External beam RT of 50-50.4 Gy (1.8 or 2 Gy per fraction) was delivered by a three-dimensional RT planning system (Eclipse; Varian Medical Systems, Palo Alto, CA, USA) using $6 \mathrm{MV}$ or $6 \mathrm{MV}$ with a $10-\mathrm{MV}$ photon. Wedge filter and field-in-field technique were used to optimize dose homogeneity. All patients with nodal involvement received regional node irradiation. Patients who underwent lumpectomy or post-mastectomy with risk factors, such as focally positive or narrow resection margins, received an additional 10-16 Gy.

\section{Patient demographics}

The study was conducted in accordance with the Declaration of Helsinki (as revised in 2013). The Institutional Review Board of Kyungpook National University Hospital (Kyungpook National University Medical Center No. 2020-03-032) approved the study and informed consent was taken from all the patients.

A total of 66 patients were divided into a radiation therapy group $(n=10)$ and a control group $(n=56)$. Patient demographics included date of surgery, age at the time of surgery, body-mass index, cancer staging, type of RT, and timing of RT. The incidence and types of complications were recorded. Patient demographic information is shown in Table 1. Aesthetic outcomes using gross photos and the incidence of complications such as seroma formation, flap necrosis, nipple-areola complex necrosis, hematoma formation, and capsular contracture development were compared between groups.

\section{Statistical analysis}

Univariate and multivariable logistic regression analysis was used to identify independent predictors of complication. In the multivariable analysis, we included the categories of the patient demographics (i.e., age, BMI, cancer staging, chemotherapy, radiation therapy timing). A crosstabulation analysis was performed for categorical data using Fisher's exact test. The log-rank test was used for all other comparisons. The $\mathrm{P}$ values of $<0.05$ were considered statistically significant. Statistical analyses were performed using IBM SPSS Version 23.0 software (IBM Corp., Armonk, NY, USA).

\section{Results}

LD flap reconstruction and implant insertion after mastectomy were performed in all 66 patients. Univariate and multivariable logistic regression analysis was used for the patient demographics and there was no significant difference among the categories in the patient demographics. In radiation group, there were four patients received additional 10 Gy and a RT of total of 60 Gy was performed, and the other 6 patients underwent conventional-normal-dosage of RT. In terms of complication rate, no statistical significance was observed between the two groups.

No significant difference in aesthetic outcomes using gross photos taken during outpatient follow-up was observed between the radiation group (Figure 1) and the control group. At 12 months postoperatively, patient satisfaction was examined using a modified Kyungpook National University Hospital Breast-Q, which consisted of 11 items in total. Each question is based on a 5 -point scale for a total of 55 points. No significant difference in patient satisfaction was observed between the two groups, with a mean score of 44.4 in the radiation group and 44.9 in the non-radiation (control) group (Table 2). No significant difference in the frequency of complications was observed between the radiation group and the control group (seroma formation, $20 \%$ versus $32 \%$, $\mathrm{P}=0.8967$; flap necrosis, $0 \%$ versus $4 \%, \mathrm{P}=1.0000$; nipple and areola complex (NAC) necrosis, $10 \%$ versus $5 \%, \mathrm{P}=0.4904$; hematoma formation, $0 \%$ versus $0 \%, \mathrm{P}=1.0000$ ); and capsular contracture development over grade 3, 10\% versus $7 \%, \mathrm{P}=0.5726$; Table 3).

Overall, 4 out of 10 patients developed complications in the radiation group, 2 (50\%) of whom developed seromas within three months of surgery, 1 (25\%) developed NAC in the fifth month after surgery, and 1 (25\%) developed capsular contracture one year postoperatively (Figure 2). A total of 27 patients developed complications in the control group. Of these, 18 patients $(66 \%)$ developed a seroma within 3 months of surgery, two patients $(7 \%)$ developed flap necrosis, three $(11 \%)$ developed NAC necrosis in the third month after surgery, one (3\%) developed capsular contracture during the tenth month after surgery, and three (11\%) developed contractures one year postoperatively. No significant differences in the timings of complications following radiation therapy were observed using the log- 
Table 1 Patient demographics

\begin{tabular}{|c|c|c|c|c|c|}
\hline Variables & \multicolumn{2}{|c|}{ Radiation group } & \multicolumn{2}{|c|}{ Non-radiation group } & $P$ value \\
\hline No. of patients & & 10 & & & \\
\hline Age at first-stage surgery, years & & & & & 0.3010 \\
\hline$\geq 0$ & 1 & 10 & 0 & 0 & \\
\hline$\geq 40$ & 7 & 70 & 27 & 48.21 & \\
\hline$\geq 50$ & 2 & 20 & 14 & 25 & \\
\hline$\geq 60$ & 0 & 0 & 8 & 14.29 & \\
\hline$>70$ & 0 & 0 & 0 & 0 & \\
\hline$\geq 18.5$ & 9 & 90 & 47 & 83.93 & \\
\hline$\geq 25$ & 1 & 10 & 8 & 14.29 & \\
\hline$\geq 30$ & 0 & 0 & 0 & 0 & \\
\hline$\geq 35$ & 0 & 0 & 1 & 1.79 & \\
\hline Cancer staging & & & & & 0.8875 \\
\hline 0 & 2 & 20 & 16 & 28.57 & \\
\hline I & 3 & 30 & 22 & 39.29 & \\
\hline II & 4 & 40 & 17 & 30.36 & \\
\hline Neoadjuvant and adjuvant (before final implant) & 0 & 0 & 0 & 0.00 & \\
\hline Neoadjuvant and adjuvant (after final implant) & 0 & 0 & 2 & 3.57 & \\
\hline Adjuvant only & 8 & 80 & 24 & 42.86 & \\
\hline Radiation therapy timing & & & & & 0.8545 \\
\hline Before final implant insertion & 0 & 0 & 0 & 0 & \\
\hline After final implant insertion & 10 & 100 & 56 & 100 & \\
\hline
\end{tabular}

BMI, body mass index.

rank test (Tables 4,5$)$.

\section{Discussion}

Advantages of immediate breast reconstruction over delayed reconstruction include outstanding aesthetic outcomes, reduced costs (being a one-stage operation), and decreased duration of breast amputation. Immediate breast reconstruction is therefore considered to improve patient psychological outcomes $(8,9)$. Fernández-Delgado 

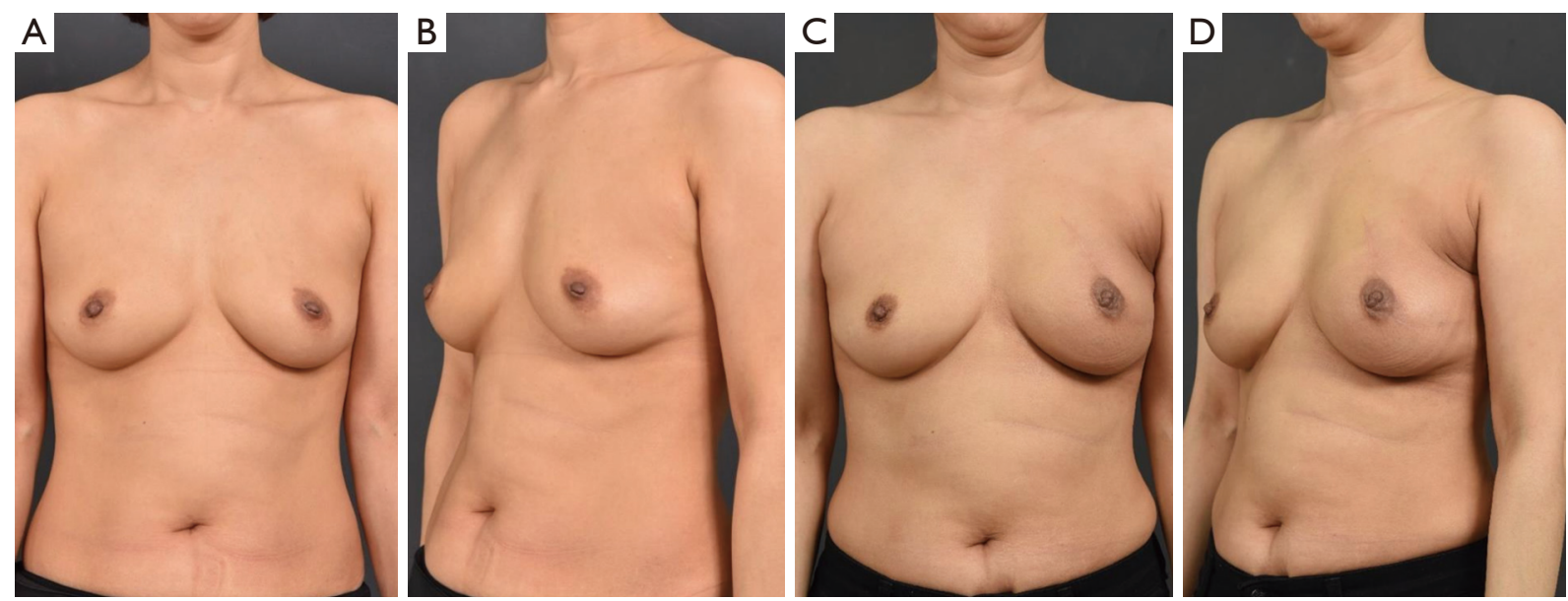

Figure 1 Breast reconstruction with latissimus dorsi and implant insertion (F/47). Post-mastectomy radiation therapy (PMRT) with 50 Gy/25 fractions. Preoperative appearances (A,B). Postoperative appearances (after PMRT) at 1 year (C,D).

Table 2 Patient satisfaction using the modified Kyungpook National University Hospital Breast-Q at 12 months postoperatively

\begin{tabular}{|c|c|c|c|c|}
\hline & Very satisfied & Radiation group & Non-radiation group & $P$ value \\
\hline $\begin{array}{l}\text { 3. Are you satisfied with the size of your breast after } \\
\text { reconstruction? }\end{array}$ & 5 & 4.4 & 4.2 & 0.284 \\
\hline $\begin{array}{l}\text { 4. Are you satisfied with the shape of your breast after } \\
\text { reconstruction? }\end{array}$ & 5 & 3.7 & 4.2 & 0.304 \\
\hline $\begin{array}{l}\text { 5. Are you satisfied with how your breasts feel after } \\
\text { reconstruction? }\end{array}$ & 5 & 4.4 & 4.1 & 0.245 \\
\hline $\begin{array}{l}\text { 6. Are you satisfied with the level of pain you had to } \\
\text { endure after reconstruction? }\end{array}$ & 5 & 4.2 & 4.1 & 0.129 \\
\hline $\begin{array}{l}\text { 8. Are you satisfied with the donor site scar (back, flank, } \\
\text { or abdomen)? }\end{array}$ & 5 & 4.0 & 4.1 & 0.101 \\
\hline $\begin{array}{l}\text { 9. Are you satisfied with the donor site pain (back, flank, } \\
\text { or abdomen)? }\end{array}$ & 5 & 3.8 & 3.6 & 0.574 \\
\hline $\begin{array}{l}\text { 10. Have you experienced a loss of confidence or } \\
\text { self-esteem after breast reconstruction? }\end{array}$ & 5 & 4 & 4.1 & 0.115 \\
\hline $\begin{array}{l}\text { 11. Are you satisfied with your sexual attractiveness after } \\
\text { breast reconstruction? }\end{array}$ & 5 & 4 & 4 & 0.497 \\
\hline
\end{tabular}


Table 3 Complications in patients undergoing LD reconstruction and implant insertion

\begin{tabular}{lccc}
\hline Variables & Radiation $(\mathrm{n}=10)$ & Non-radiation $(\mathrm{n}=56)$ & $\mathrm{P}$ value \\
\hline Seroma & $2(20 \%)$ & $18(32 \%)$ & 0.8967 \\
Flap necrosis & $0(0 \%)$ & $2(4 \%)$ & 1 \\
NAC necrosis & $1(10 \%)$ & $3(5 \%)$ & 0.4904 \\
Hematoma & $0(0 \%)$ & $0(0 \%)$ & 1 \\
Capsular contracture & $1(10 \%)$ & $4(7 \%)$ & 0.5726 \\
No complication & $6(60 \%)$ & $29(51 \%)$ & 0.7286 \\
\hline
\end{tabular}

NAC, nipple and areola complex.
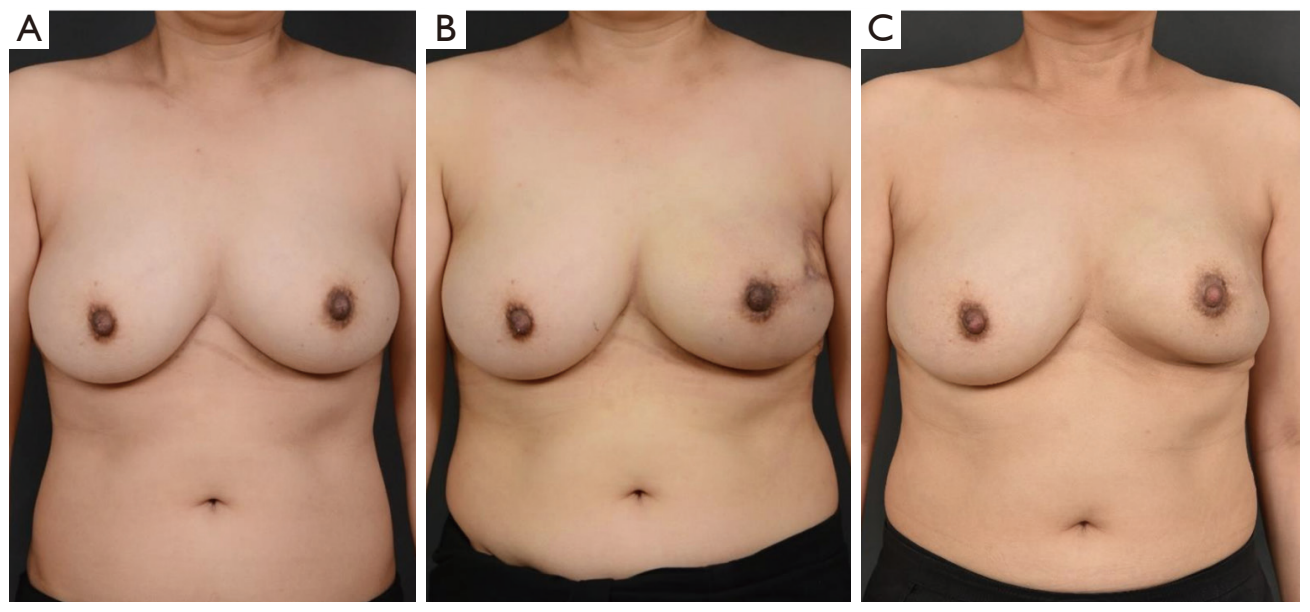

Figure 2 Breast reconstruction with latissimus dorsi and implant insertion (F/48). Post-mastectomy radiation therapy (PMRT) with 50 Gy/25 fractions. Capsular contracture is not observed at one month postoperatively (B) but can be seen at 2 years postoperatively (C). Postoperative photo findings at day 3 (A), one month (B, after PMRT), and at 2 years (C, after PMRT).

Table 4 Time intervals to the first complication in the radiation group

\begin{tabular}{|c|c|c|c|c|c|c|c|c|c|c|c|c|}
\hline Complication & \multicolumn{10}{|c|}{ Time (months) } & Total & $P$ value \\
\hline Seroma & 2 & 0 & 0 & 0 & 0 & 0 & 0 & 0 & 0 & 0 & 2 & $0.0475^{\star}$ \\
\hline Flap necrosis & 0 & 0 & 0 & 0 & 0 & 0 & 0 & 0 & 0 & 0 & 0 & 1 \\
\hline NAC necrosis & 0 & 1 & 0 & 0 & 0 & 0 & 0 & 0 & 0 & 0 & 1 & 0.5872 \\
\hline Capsular contracture & 0 & 0 & 0 & 0 & 0 & 0 & 0 & 0 & 0 & 1 & 1 & 0.7066 \\
\hline No complication & & & & & & & & & & & 6 & \\
\hline
\end{tabular}

${ }^{\star}, \mathrm{P}<0.05$. NAC, nipple and areola complex. 
Table 5 Time intervals to the first complication incidence in the non-radiation group

\begin{tabular}{|c|c|c|c|c|c|c|c|c|c|c|c|c|}
\hline Complication & \multicolumn{10}{|c|}{ Time (months) } & Total & $P$ value \\
\hline Seroma & 18 & 0 & 0 & 0 & 0 & 0 & 0 & 0 & 0 & 0 & 18 & $0.0475^{*}$ \\
\hline Flap necrosis & 2 & 0 & 0 & 0 & 0 & 0 & 0 & 0 & 0 & 0 & 2 & 1 \\
\hline NAC necrosis & 3 & 0 & 0 & 0 & 0 & 0 & 0 & 0 & 0 & 0 & 3 & 0.5872 \\
\hline Capsular contracture & 0 & 0 & 0 & 0 & 0 & 0 & 0 & 1 & 0 & 3 & 4 & 0.7066 \\
\hline No complication & & & & & & & & & & & 29 & \\
\hline
\end{tabular}

*, $\mathrm{P}<0.05$. NAC, nipple and areola complex.

et al. (9) reported $94.77 \%$ of patients preferred immediate breast reconstruction over the delayed approach. Immediate breast reconstruction is also associated with good outcomes in patients receiving radiation therapy; however, surgical methods and timing remain controversial.

PMRT is one of many factors known to influence the aesthetic outcomes and complications of reconstruction after a mastectomy, with some authors suggesting breast reconstruction using implants should not be performed in breast cancer patients for whom RT is planned (10-12). Many studies have reported that radiation therapy after implant surgery is associated with an increased frequency of complications, including the need for revision, capsular contracture, implant exposure, implant rupture, and implant removal (13). Moreover, autologous-based reconstruction can be affected by radiation therapy, and adverse effects may occur as a result (7).

Similar length of hospital stay and need for additional operations have been reported between patients undergoing both the LD flap procedure and implant insertion compared to patients undergoing only LD flap reconstruction (1). It is generally recognized that the use of additional implants for insufficient volume supplementation is safe and results in increased patient satisfaction $(1,14)$.

Berthet et al. evaluated breast consistency following surgery in 154 patients who underwent LD flap reconstruction, with $93.1 \%$ of patients rating outcomes as "very good" or "good" in the irradiated group and $82.7 \%$ in the non-irradiated group; however, the difference was not statistically significant (8). The low rate of fat necrosis following RT observed in LD flaps may be explained by the lower proportion of fatty tissue compared to abdominal wall flaps or other autologous tissues; however, the exact mechanism underlying this observation is yet to be elucidated (8).

The present study evaluated radiation therapy as an adjunct to LD flap reconstruction and implant insertion, techniques considered important methods for breast reconstruction. Although an increased frequency of complications, particularly capsular contracture, has been reported with radiation therapy in patients undergoing only implant insertion $(15,16)$, the present study found no increase in complications with radiation therapy in patients undergoing both LD flap reconstruction and implant insertion. In addition, no differences in aesthetic outcomes were observed between the radiation and control groups.

LD flap tissue appears to be more protected from radiation than other autologous tissues used for reconstruction, such as abdominal wall flap tissue. Further, healthy tissues adjacent to implants appear to be protected from radiation injury after implant insertion (7).

We also observed that radiation had no effect on the timing of complication development, with no statistically significant difference observed between patients who did and did not receive radiation. Most complications developed within three months of surgery except for capsular contractures, where the majority of cases developed at one year postoperatively.

The present study had several limitations. It had a limited number of patients, thereby limiting statistical power. In addition, the duration of the outpatient followup was limited, with many patients lost to follow-up at one year postoperatively.

The present study adds important information on breast reconstruction. The findings of this study demonstrate that LD flap reconstruction with implant insertion is a comparatively safe method even among patients receiving radiation. These surgical procedures can be performed 
safely and with acceptable aesthetic outcomes. These findings indicate LD reconstruction and implant insertion can be performed simultaneously to avoid deformity and asymmetry due to lack of volume.

\section{Conclusions}

We demonstrate that radiation therapy is relatively safe in patients undergoing $\mathrm{LD}$ reconstruction with implant insertion due to a lack of LD flap volume. Furthermore, these surgical procedures result in excellent aesthetic outcomes.

\section{Acknowledgments}

We thank Enago (https://www.enago.jp/) for editing this manuscript.

Funding: None.

\section{Footnote}

Reporting Checklist: The authors have completed the STROBE reporting checklist. Available at https://dx.doi. org/10.21037/gs-21-219

Data Sharing Statement: Available at https://dx.doi. org/10.21037/gs-21-219

Conflicts of Interest: All authors have completed the ICMJE uniform disclosure form (available at https://dx.doi. org/10.21037/gs-21-219). The authors have no conflicts of interest to declare.

Ethical Statement: The authors are accountable for all aspects of the work in ensuring that questions related to the accuracy or integrity of any part of the work are appropriately investigated and resolved. The study was conducted in accordance with the Declaration of Helsinki (as revised in 2013). The Institutional Review Board of Kyungpook National University Hospital (Kyungpook National University Medical Center No. 2020-03-032) approved the study and informed consent was taken from all the patients.

Open Access Statement: This is an Open Access article distributed in accordance with the Creative Commons Attribution-NonCommercial-NoDerivs 4.0 International License (CC BY-NC-ND 4.0), which permits the non- commercial replication and distribution of the article with the strict proviso that no changes or edits are made and the original work is properly cited (including links to both the formal publication through the relevant DOI and the license). See: https://creativecommons.org/licenses/by-nc-nd/4.0/.

\section{References}

1. Leuzzi S, Stivala A, Shaff JB, et al. Latissimus dorsi breast reconstruction with or without implants: A comparison between outcome and patient satisfaction. J Plast Reconstr Aesthet Surg 2019;72:381-93.

2. Early Breast Cancer Trialists' Collaborative Group (EBCTCG); Darby S, McGale P, et al. Effect of radiotherapy after breast-conserving surgery on 10year recurrence and 15-year breast cancer death: metaanalysis of individual patient data for 10,801 women in 17 randomised trials. Lancet 2011;378:1707-16.

3. EBCTCG (Early Breast Cancer Trialists' Collaborative Group); McGale P, Taylor C, et al. Effect of radiotherapy after mastectomy and axillary surgery on 10-year recurrence and 20-year breast cancer mortality: metaanalysis of individual patient data for 8135 women in 22 randomised trials. Lancet 2014;383:2127-35.

4. Frasier LL, Holden S, Holden T, et al. Temporal Trends in Postmastectomy Radiation Therapy and Breast Reconstruction Associated With Changes in National Comprehensive Cancer Network Guidelines. JAMA Oncol 2016;2:95-101.

5. Jagsi R, Momoh AO, Qi J, et al. Impact of Radiotherapy on Complications and Patient-Reported Outcomes After Breast Reconstruction. J Natl Cancer Inst 2018;110:157-65.

6. Lujan-Hernandez J, Chin MS, Perry DJ, et al. Increasing Fat Graft Retention in Irradiated Tissue after Preconditioning with External Volume Expansion. Plast Reconstr Surg 2020;145:103-12.

7. Spear SL, Boehmler JH, Taylor NS, et al. The role of the latissimus dorsi flap in reconstruction of the irradiated breast. Plast Reconstr Surg 2007;119:1-9.

8. Berthet G, Faure C, Dammacco MA, et al. Tolerance of latissimus dorsi in immediate breast reconstruction without implant to radiotherapy. J Plast Reconstr Aesthet Surg 2018;71:15-20.

9. Fernández-Delgado J, López-Pedraza MJ, Blasco JA, et al. Satisfaction with and psychological impact of immediate and deferred breast reconstruction. Ann Oncol 2008;19:1430-4. 
10. Evans GR, Schusterman MA, Kroll SS, et al. Reconstruction and the radiated breast: is there a role for implants? Plast Reconstr Surg 1995;96:1111-5; discussion 1116-8.

11. Forman DL, Chiu J, Restifo RJ, et al. Breast reconstruction in previously irradiated patients using tissue expanders and implants: a potentially unfavorable result. Ann Plast Surg 1998;40:360-3; discussion 363-4.

12. Vandeweyer E, Deraemaecker R. Radiation therapy after immediate breast reconstruction with implants. Plast Reconstr Surg 2000;106:56-8; discussion 59-60.

13. Ascherman JA, Hanasono MM, Newman MI, et al. Implant reconstruction in breast cancer patients

Cite this article as: Kim YH, Lee JS, Park J, Lee J, Park HY, Yang JD. Aesthetic outcomes and complications following postmastectomy radiation therapy in patients undergoing immediate extended latissimus dorsi flap reconstruction and implant insertion. Gland Surg 2021;10(7):2095-2103. doi: 10.21037/gs-21219 treated with radiation therapy. Plast Reconstr Surg 2006;117:359-65.

14. Feng J, Pardoe CI, Mota AM, et al. Two-Stage Latissimus Dorsi Flap with Implant for Unilateral Breast Reconstruction: Getting the Size Right. Arch Plast Surg 2016;43:197-203.

15. Yun JH, Diaz R, Orman AG. Breast Reconstruction and Radiation Therapy. Cancer Control 2018;25:1073274818795489.

16. Spear SL, Onyewu C. Staged breast reconstruction with saline-filled implants in the irradiated breast: recent trends and therapeutic implications. Plast Reconstr Surg 2000;105:930-42. 